\title{
Study on Development of Novel Mg-Based Alloys by Rapid Solidification Technology of Twin Roll Casting
}

\author{
Haijian Wang ${ }^{1, a}$, Zhipu Pei ${ }^{1, b}$ and Dongying Ju, ${ }^{2, c^{*}}$ \\ ${ }^{1}$ Department of Material Science and Engineering, Saitama Institute of Technology Fusaiji1690, \\ Fukaya, Saitama 369-0293, Japan \\ 2Department of Material Science and Engineering, University of Science and Technology Liaoning, \\ No.185 Qianshan Mid-Road, Anshan, Liaoning,114051,China \\ ae7002qox@sit.ac.jp, bpeizhipu@gmail.com, dyju@sit.ac.jp
}

Keywords: Metallic glasses; rapid solidification; quasi-amorphous; GFA.

Abstract. Mg-based alloys were prepared by rapid solidification of twin $\mathrm{ro}^{\mathrm{y}}$-asth (TRC), hen microstructure and element distribution were investigated by means of scar ing e ectron icr oscope (SEM) and electron probe micro analyzer (EPMA). The analyze micros ure sucr. sta structure of Mg-based alloys were characterized by X-ray diffraction and tra iss electro microscope (TEM). The designed Mg-rare-earth (RE) alloy with quasi-amorph and fine ta ine dual -phase microstructure was produced by rapid solidification using $\mathrm{T}^{\mathrm{C}} \mathrm{C}$ p ess. The apid solidification process was realized by a faster casting speed and a thinner ro a with anther addional devices and vacuum environment. EPMA results and TEM analys $s$ show that the $q$ si-amorphous phase had a high concentration in $\mathrm{Al}$ and $\mathrm{RE}$ element. A quasi-am phous phase surrounded by dendrites phase and normal crystals in the middle of Mg-Re alloy was o erved .

\section{Introduction}

Metallic glasses are currently the focus of inte se eso. $n$ in the international metals community due to their special microstructure andapropertic [1]. However, it is still a mystery about those questions on the development, stry cture d phys al properties of this new phase. Glass-forming systems have been found in all o or $b$ mding classes, including covalent, ionic, van der Waals, hydrogen bond, and metallic ${ }^{-}$i tomms of metallic glasses (MGs) needs extremely high cooling rates (on the order $10^{5}$ to 10 ) due to the non-directional nature of metallic bonds and the fact that metals ar con ised of dividual spherical atoms, as opposed to non-spherical compounds such as $\mathrm{S}_{2}$ and ot quickly into lowe enery configu. cons or crystalline phases, a higher cooling rate is required to avoid crystalliz

In 1970, Twin asting developed Chen Miner [4]. To date, this technique in producing metallic glass ribbons is almos imite to labo, ory scale studies; however TRC is an available process for producing amorp a with a wide range of cooling rates. In this work, we focused on developing a new kind Mg-based alloy with the quasi-amorphous phase. Considering its application, we proposed a competitive method to produce the new material in sheet form. It considered as an efficient mass-production technique.

\section{Alloy Composition Design}

The glass forming ability rules in Mg-based materials. Mismatch entropy normalized by Boltzmann constant $\left(S_{\zeta} / k_{B}\right)$ based on hard sphere model and mixing enthalpy $(\Delta H)$ based on regular solution model were expressed as a function of composition in multicomponent systems by Takeuchi [5] and Inoue [6]. Atomic size distribution plots for the glass forming ability (GFA) were proposed by Senkov [7]. It was found that a concave upward distribution correlates so strongly with improved glass forming ability. Mg-based alloys are deviate from this trend. This is another exception of Mg-based materials. 
Compositions of the alloys. In order to retain an expect structure from the melt, (1) appropriate quenching techniques must be applied, and (2) careful alloy selection must be made. Considering from the topological aspect, atomic radii of candidate alloying elements for magnesium alloys are listed in Table 1. Atomic size differences between the alloying elements and magnesium (aluminum) are also calculated which symbolized by $\mathrm{ASD}_{\mathrm{Mg}}\left(\mathrm{ASD}_{\mathrm{Al}}\right)$.

Table 1. Atomic radius of some common elements used in Mg alloys.

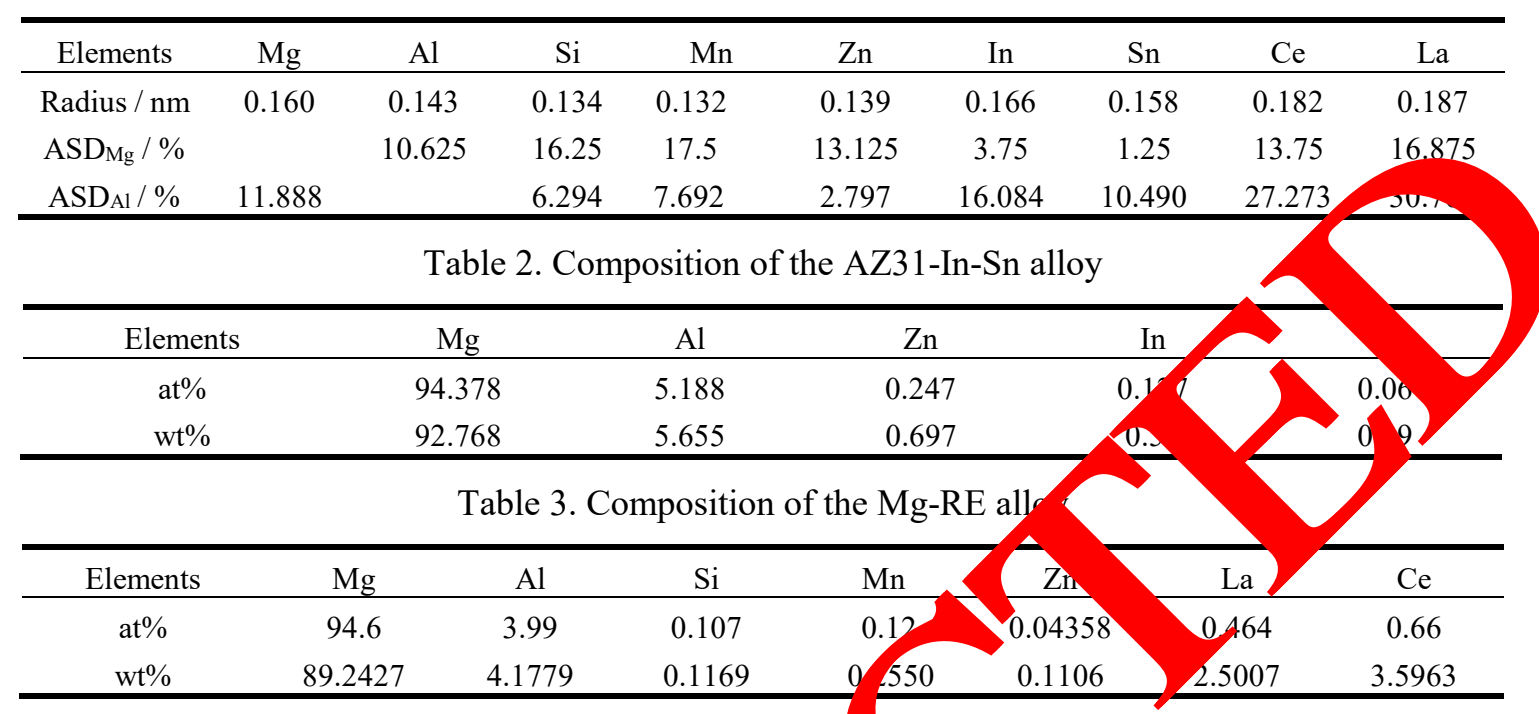

AZ31B is a popular wrought magnesium alloy ed in ma y aerospace and automotive applications. However, its widespread apply is hinderea the or corrosion resistance and low ductility. As Sn has the potential of improve co cistance and ductility of Mg-Al alloy [8], In is used in $\mathrm{Mg}$ battery materials [9]. With the pur ose on $\mathrm{Ag}^{2}$ to find out whether it can form an amorphous phase contained material through the pid scidification of TRC method in the current study, AZ31-In-Sn alloy was studie ${ }^{\prime}$ in 4 work. Z31 alloy was adopted as a material for contrast.

Rare earth (RE) element has arac ristic of o-called-scavenger effect in magnesium alloys [10]. Impurity elements cou' orn ss camanc intermetallic compounds with RE. In order to improve the corrosion re tance of product, lanthanum and cerium were adopted in our designment. The compe tion mer melteg high frequency induction melting are listed in Table 2 and Table 3 and the r nompo were tested by X-ray fluorescence (XRF) analysis method.

3. Experimea ind orscu sion

Microstry cha teric ac. In order to achieve a faster cooling rate by the TRC process, we set the spe of rolls a Jrpm, Considering the separating force generated during casting, a metal block se the moving roll side to form a supporting force, to make a narrow roll gap during casting po ss as much as possible.

The SEM ge of the AZ31 alloy strip with thickness of $1.2 \mathrm{~mm}$ is shown in Fig. 1(a). It has an average seconary dendrite arm spacing (DAS) of about $0.7 \mu \mathrm{m}$. It is found that the $\mathrm{Mg}$ is uniformly distributed in this area due to the very fine dendrite. It is quite difficult to obtain a glassy state of AZ31 alloy even though we set a faster cooling rate hardly. Fig. 1(b) shows the cross-sectional SEM image obtained near the sliced surface. There are a number of fine grains having sizes of $0.5 \sim 2 \mu \mathrm{m}$ due to the effect of chilling. Although a narrow DAS at the center of the slice and fine grains near the surface of the slice formed during the rapid cooling process, a crystalline phase is observed under such a limit condition of the twin roll caster. SEM images of Mg-RE alloy sheet are shown in Fig. 1(c). The microstructure mainly characterized by fine equiaxed grains and dendrites with closely spaced secondary dendrite arms. Meanwhile, there are some special areas having no appreciable crystalline features indicated by the red arrows. We name them quasi-amorphous solids, and the structure was 
characterized by the following X-ray diffraction (XRD) and transmission electron microscopy (TEM) analysis. Critical cooling rate for forming metallic glass with the composition of the quasi-amorphous phase regions is lower than that of the crystalline phase regions, in other words, the glass forming ability (GFA) of the Mg-RE is higher.
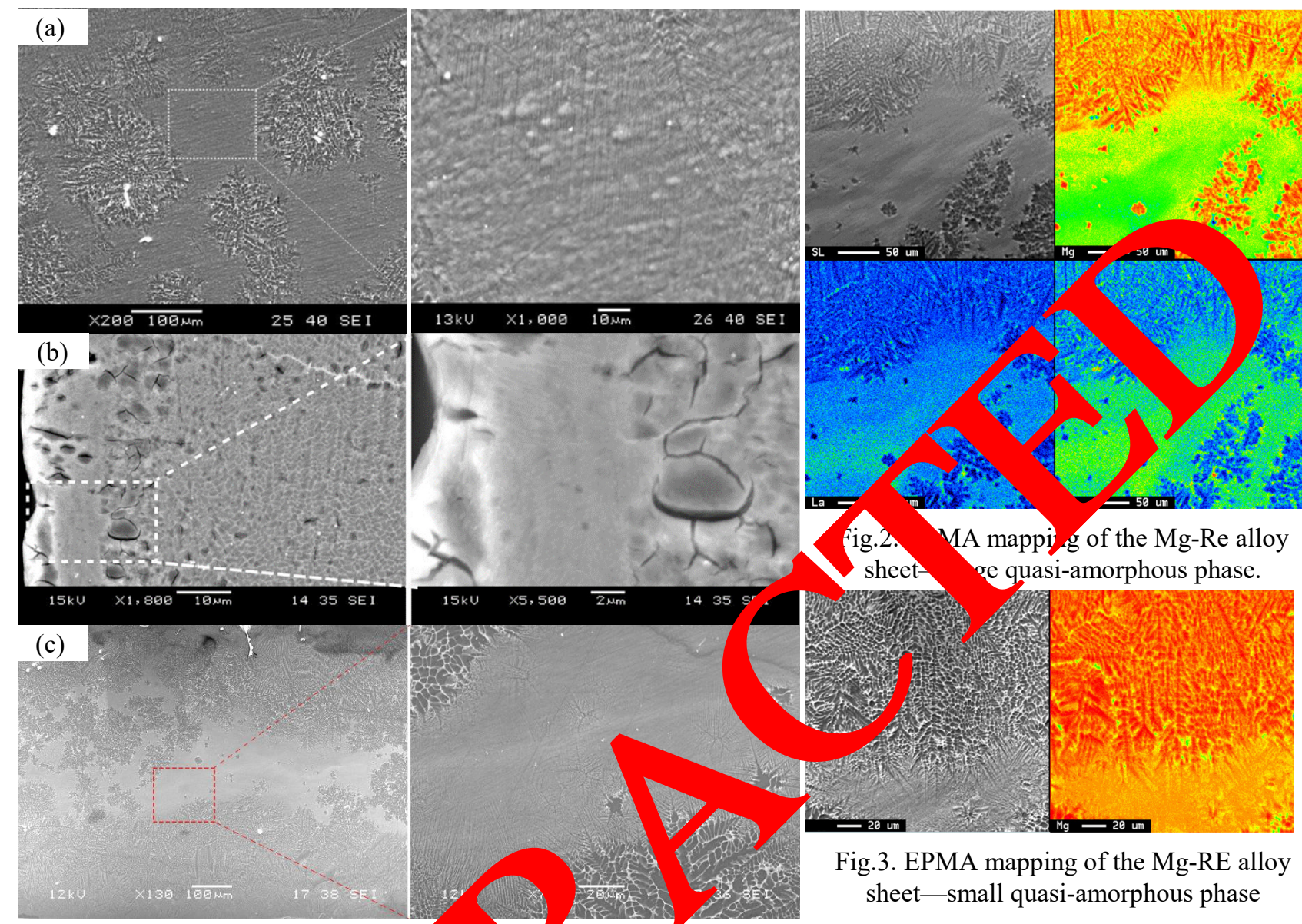

ig.2. MA mappi, go the Mg-Re alloy

Fig.1. SEM image of as-cast (a) AZZ1 a $(b-2)$

(c) Mg-Re alloy shee Alr wans

section.

Elements distribution ma f the Mg RE alloy sheet sample were obtained by electron probe micro-analysis (EPMN, as sh in Fig. 2 and 3. On the one hand, element segregation exists between the crysta ine nhase matr and the quasi-amorphous phases. On the other hand, it is shown that grain boun $\mathrm{V}$ seg ation forms easily as the atomic size difference (ASD) between Mg and alloying element $\mathrm{ger}$ thar $0 \%$. As the atomic sizes between $\mathrm{Mg}-\mathrm{Al}, \mathrm{Mg}-\mathrm{La}, \mathrm{Mg}-\mathrm{Ce}, \mathrm{Al}-\mathrm{La}$ and Al-Ce are diffe do dritic segregations also generate in the crystalline matrix.

Bas on th EPMA alyses of Fig. 2 and 3, microstructure characteristic of the quasi-amorphous phase a its ings are illustrated in Fig. 4. At zone A shows the crystalline matrix with fine equiaxed $\mathrm{ns}_{\mathrm{s}}$ and dendrites, segregation of elements occurs at the grain boundaries and dendrite spaces. At $z$ B where fine dendrites with closely spaced secondary dendrite arms distributed around a large quasi-amorphous phase, element segregation of this zone decreased due to the fine dendrites. Elements uniformly distribute the quasi-amorphous phase as indicated by zone C. It contains a low amount of $\mathrm{Mg}$ comparing to the crystalline matrix. Contrarily, the amount of $\mathrm{Al}$ and $\mathrm{La}$ is relatively large. Although a few nuclei inside the quasi-amorphous phase, their growths are restricted and the final sizes are less than $20 \mu \mathrm{m}$. 


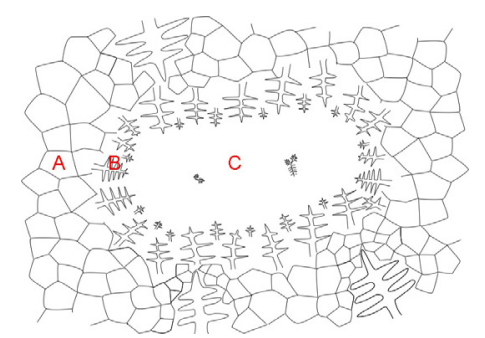

Fig.4. Schematic illustration of microstructure characteristic of the quasi-amorphous phase and its surroundings.

\section{X-ray diffraction analysis}
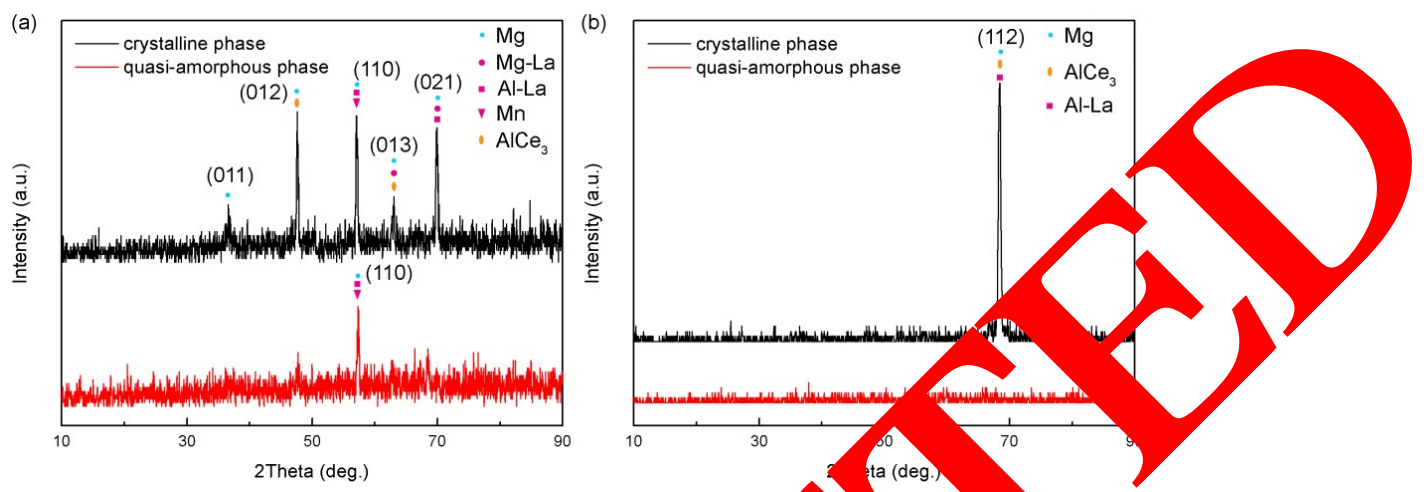

Fig.5. $\mu$-XRD patterns of the as-cast sheet mid-thickness: (a) small

(b) large quasi-amorphous phase witho

Micro-XRD ( $\mu$-XRD) patterns obtained from the as- $\mathrm{tg}-\mathrm{RE}$ alloy sheet sample are shown in Fig. 5 Several diffraction peaks appear in the profile obta from ${ }^{\prime}$ e crystalline phase. A weak peak in the quasi-amorphous phase at $2 \theta=57.4^{\circ}$, collimated incident beam $(\Phi=0.03 \mathrm{~mm})$ and son 01 rotline phase information was mixed in it. Another reason of the peak is the find dendri cexisted in the large quasi-amorphous solids, as shown in Fig. 1(c). In Fig. 5(b), the patter obtained from the crystalline phase exhibits only one peak at $2 \theta=68.4^{\circ}$. This may ue to the detec on area contains a single crystal and a small quasi-amorphous phase.

TEM analysis. TEM imag of the as st Mg-RE alloy shown in Fig. 6. SAED patterns are also shown in the insets. In $\mathrm{Cl}$ (a) the gi consists of $\mathrm{Mg}$ crystal having a grain size of $5 \mu \mathrm{m}$. According to model we ropos in Fig. 4, the $\mathrm{Mg}$ crystal corresponds to the zone A. The very fine grains with average gram size of 0.11 are observed in Fig. 6(a) and (b). These area corresponding to the zone $\mathrm{B}$ in $\mathrm{Fi}^{4}$ 4. The AED patern reveal that these grains are Mg-La phase.

TEM image o1 ain and s grain boundary and the corresponding SAED patterns are shown in Fig. 6(d). Mg gro vize, $7 \mu \mathrm{m}$. Near the grain boundary, there also an amount of very fine grains with si e of $0, \mu \mathrm{m}$. Th grains are identified as Mg-La and Al-La phases. It indicates that these two phases cip zone B an in Fig. 4 correspond to Figs. 6(e) and (f). In the zone B, it contains fine dendrites feature. In the zone shows a poor crystallinity. Some diffraction spots appears in the SAED patterns, because the size quasi-amorphous phase was small or the specimen damaged due to the electron irradiation.

\section{Conclusion}

The designed Mg-RE magnesium alloy with quasi-amorphous phase and fine crystalline phase dual phase microstructure produced with the rapid solidification of TRC process. The rapid solidification process realized by a faster casting speed and a thinner roll gap without additional devices and vacuum environment. EPMA results and TEM analyses show that the quasi-amorphous phase has a 
high concentration in $\mathrm{Al}$ and $\mathrm{RE}$ element, which results in the fact that the Mg-RE alloy had a better GFA.
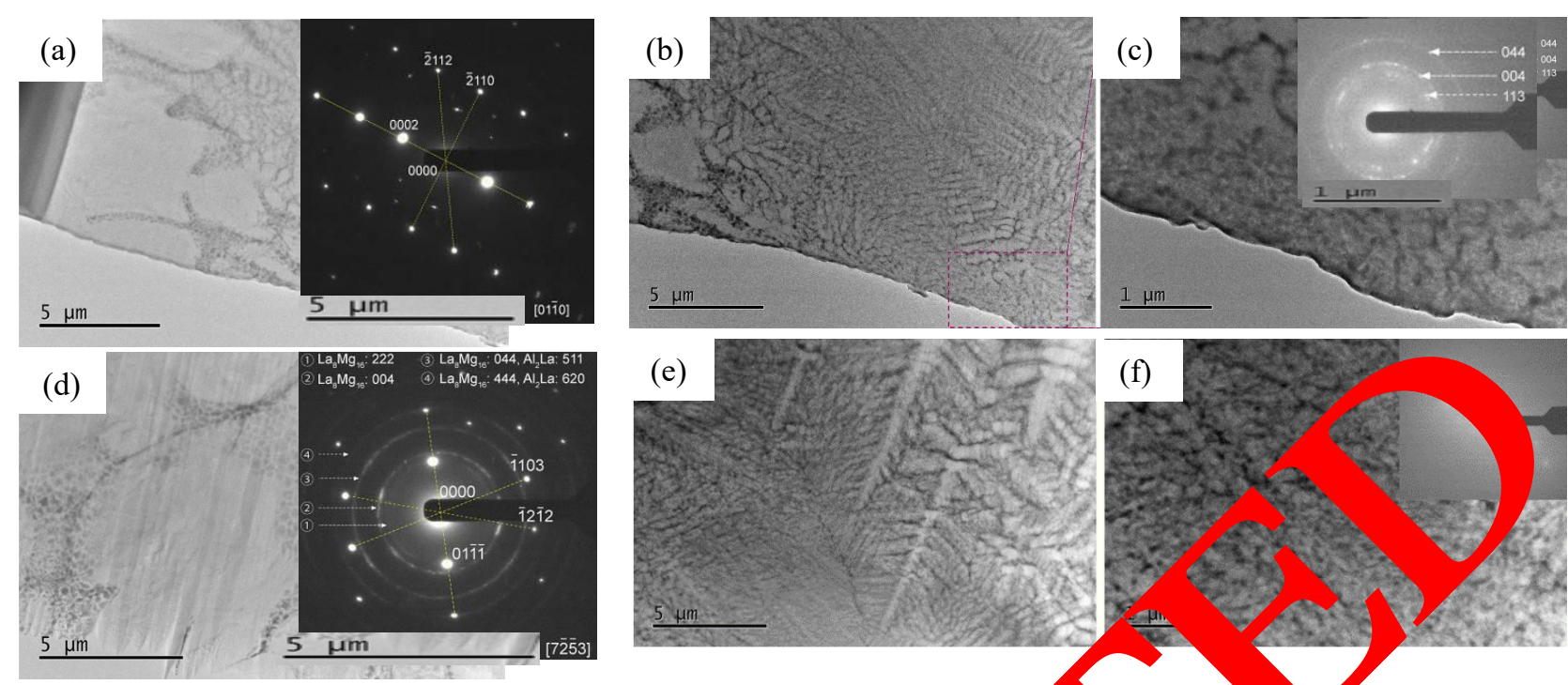

(f)

Fig.6. TEM image of the as-cast Mg-RE alloy. Inset, SAED patter o grain - (a M-RE alloy;

(b) (c) $\mathrm{La}_{8} \mathrm{Mg}_{16}$ phase; (d) $\mathrm{Mg}$ grain and La-Mg, Al-La phase at graí found. (e) fine Cendrites; (f) quasi-

\section{Acknowledgement}

This work was supported by Cooperative Research and D 1 opme it Center for Advanced Materials (CRDAM) funded by the Institute for Materials arch (IMm), Tohoku University (Project Number 18G0042).

\section{References}

[1] Green A L, Ma E. Bulk Neta G the Cutting Edge of Metals Research [J]. MRS Bulletin, 2007, 32(08): 611- 19 .

[2] Susanne S. Bulk me alli asses [J]. urnal of Physics: Condensed Matter, 2001, 13(34): 7723. [3] Arata E R. Hig'mperatur Mltyahigh-gravity centrifugal processing of metallic melts [D]; Citeseer, 2008.

[4] Chen H S, M E. A A pid Quenching Technique for the Preparation of Thin Uniform Films of Amorpb Solia Re rew of Scientific Instruments, 1970, 41(8): 1237-1238.

[5] T suchi 1, Inoue A. Calculations of Mixing Enthalpy and Mismatch Entropy for Ternary Amorph Materials Transactions, JIM, 2000, 41(11): 1372-1378.

[6] Inoue A. ${ }^{1 k}$ amorphous and nanocrystalline alloys with high functional properties [J]. Materials Science and E, gineering: A, 2001, 304-306(1): 1-10.

[7] Senkov O N, Miracle D B. Effect of the atomic size distribution on glass forming ability of amorphous metallic alloys [J]. Materials Research Bulletin, 2001, 36(12): 2183-2198.

[8] She J, Pan F, Zhang J, et al. Microstructure and mechanical properties of Mg-Al-Sn extruded alloys [J]. Journal of Alloys and Compounds, 2016, 657(1): 893-905.

[9] Murgia F, Weldekidan E T, Stievano L, et al. First investigation of indium- based electrode in Mg battery [J]. Electrochemistry Communications, 2015, 60(1): 56-59.

[10] Liu W, Cao F, Chang L, et al. Effect of rare earth element Ce and La on corrosion behavior of AM60 magnesium alloy [J]. Corrosion Science, 2009, 51(6): 1334-1343. 